\title{
Graphite nodules features identifications and damaging micromechanims in ductile irons
}

\author{
Alberto De Santis, Daniela Iacoviello \\ Sapienza Università di Roma, Dipartimento di Ingegneria Informatica, Automatica e Gestionale Antonio Ruberti, Italy
}

\author{
Vittorio Di Cocco, Francesco Iacoviello \\ Università di Cassino e del Lazio Meridionale, DICeM, via G. Di Biasio 43, 03043 Cassino (FR), Italy \\ iacoviello@unicas.it
}

\begin{abstract}
Ductile irons mechanical properties are strongly influenced by the metal matrix microstructure and on the graphite elements morphology. Depending on the chemical composition, the manufacturing process and the heat treatments, these graphite elements can be characterized by different shape, size and distribution. These geometrical features are usually evaluated by the experts visual inspection, and some commercial softwares are also available to assist this activity. In this work, an automatic procedure based on an image segmentation technique is applied: this procedure is validated not only considering spheroidal graphite elements, but also considering other morphologies (e.g. lamellae).
\end{abstract}

KEYWORDS. Ductile irons; Damaging micromechanisms; Image segmentation; Level sets.

\section{INTRODUCTION}

$\mathrm{D}$ amaging micromechanisms evolution in ductile cast irons (DCIs) is strongly influenced by the matrix microstructure and by the graphite nodules morphology. Considering recent experimental results [1-6], the role played by the graphite nodules is not merely connected to a matrix-graphite debonding mechanism, followed by voids nucleation and growth as described in [7-10]. Graphite nodules damaging micromechanisms can be classified as follows:

- Graphite - matrix debonding (Fig.1 - Video 1);

- "Onion-like" mechanism (Fig. 2 - Video 2);

- Crack initiation and propagation in the "nodule core" (Fig. 3 - Video 3).

Fig. $1-3$ (and Videos 1-3) are obtained according to the procedure described in [1-6] and refers to a fully pearlitic DCI. In order to obtain the videos, a "step by step" testing procedure allowed "in situ" scanning electron microscope (SEM) observations and a commercially available morphing software was used (at least, 10 step for each video).

The pearlitic matrix - graphite nodule debonding (Fig. 1 - V1) is characterized by a debonding nucleation that is obtained for loading conditions that corresponds to the elastic stage: debonding nucleation is evident in the nodule "pole" and becomes more and more evident with the increase of the macroscopic deformation.

The "onion -like" mechanism is probably connected to a mechanical properties gradient in the graphite nodule, probably due to the graphite nodule nucleation and growth mechanisms. This gradient has been observed in ferritic DCI by means of nano indentation tests [11]. The "onion-like" mechanism nucleates already corresponding to a stress value of about 600 $\mathrm{MPa}$ (elastic stage): some cracks nucleate on the higher-left side of the nodule (Fig. 2 - V2). The increase of the macroscopic deformation implies a propagation of the first cracks and the nucleation of new cracks, allowing to define a 
both sort of "nodule core" (obtained during the solidification from the melt) and a "graphite shield" (obtained by means of Carbon solid diffusion through the austenite shield during the DCI cooling) that is connected to the pearlitic matrix.
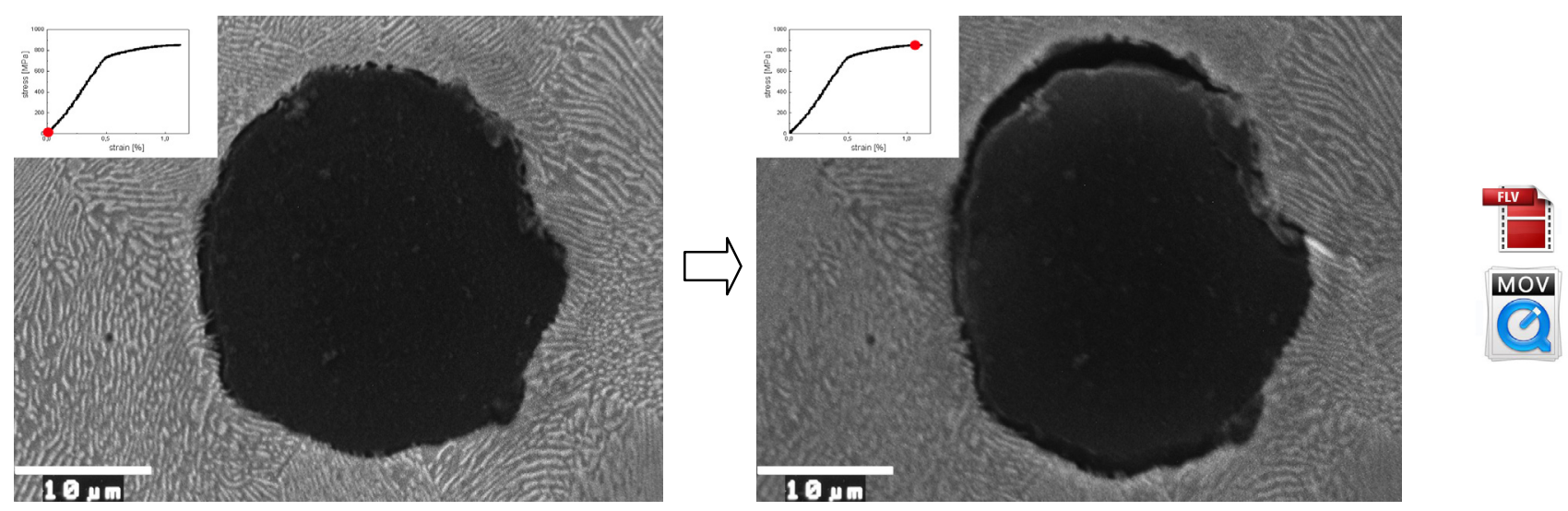

Figure 1: Pearlitic DCI: graphite - matrix debonding [V1].
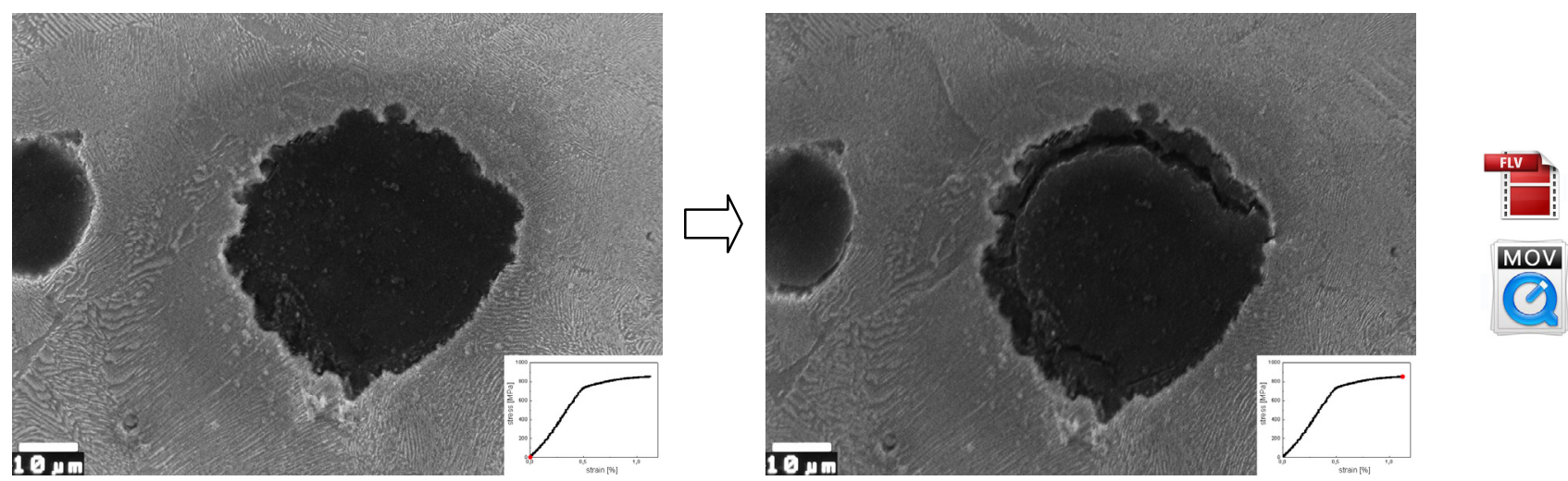

Figure 2: “Onion-like” mechanism [V2].

The last mechanism (Fig. 3 - V3) is the crack nucleation and growth corresponding to the nodule center, probably the nodule nucleation site. Always corresponding to a loading value that is lower than DCI yield strenght, a crack nucleates in the nodule center: the macroscopic deformation increases, implying both a crack propagation inside the graphite element (with a crack path that is orthogonal to the loading direction) and an increase of the crack opening. Corresponding to the higher deformation level, both the "onion-like" and the matrix - graphite debonding mechanisms are also active.
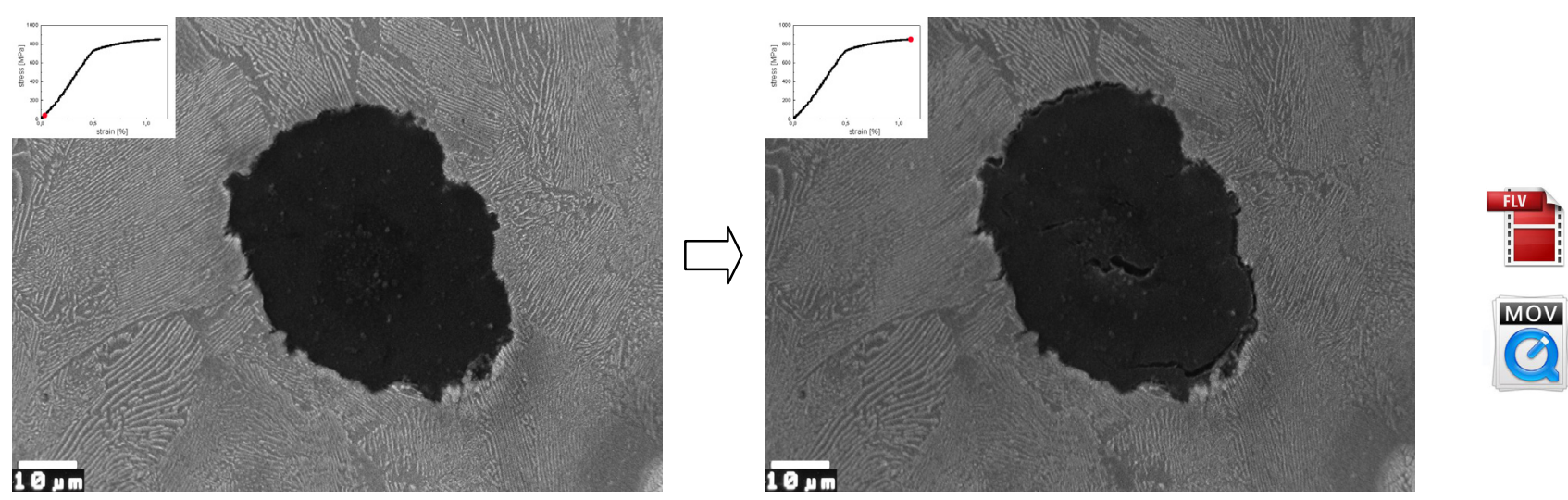

Figure 3: Crack initiation and propagation in the "nodule core" [V3]. 
The importance of the different damaging micromechanisms is influenced by the matrix microstructure, but it is worth to note that, considering ferritic-pearlitic ductile irons, matrix - graphite nodule debonding is not the most important damaging micromechanism.

Focusing the role played by graphite elements in a completely different cast iron (a fully pearlitic flake cast iron), the flaky graphite tends to open in the middle and a void appears inside, considering both graphite elements orientated perpendicular and parallel to the loading direction [12]. The load increase implies an increase of the cast iron internal damage, with a graphite elements - pearlitic matrix debonding. This mechanism is more evident with the graphite elements oriented perpendicular to the loading direction. Furthermore, a microplasticity at graphite tips is observed in the pearlitic matrix (Fig. 4).

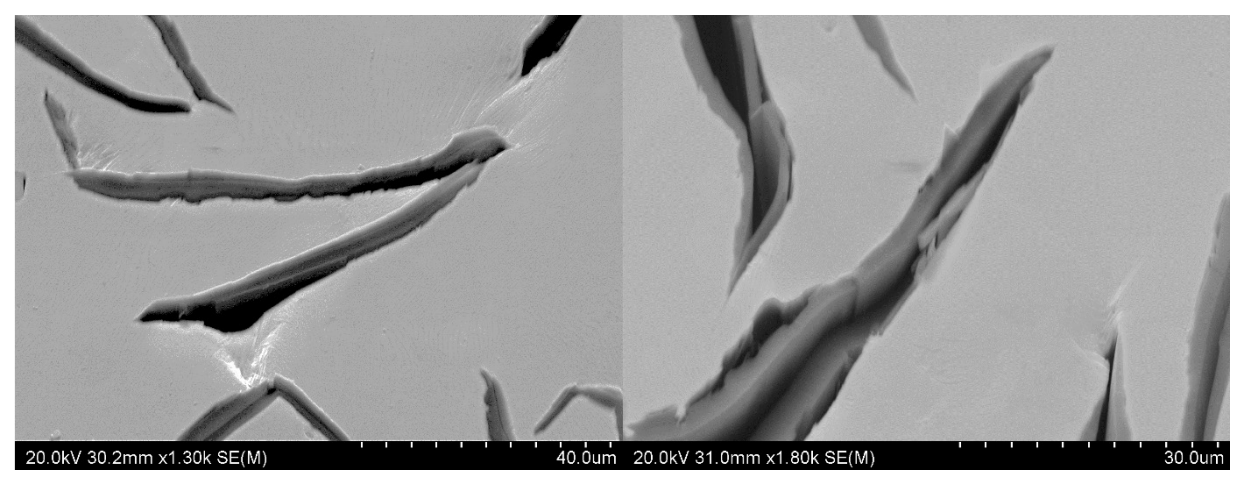

Figure 4: Damaging micromechanims in a fully pearlitic flake cast iron [12].

Considering the results in Fig.-1-4 and in Videos 1-3, it is evident the strong influence of the graphite nodules morphology on the damaging micromechanisms: the visual qualitative approach followed in the EN ISO standard [13] does not seem to be sufficient to fully characterize the graphite elements in a cast iron and therefore a quantitative approach seems to be necessary.

Images obtained by means of LOM, despite a good visual appearance, are represented by a quite irregular signal due to various kind of degradations stemming from the acquisition process: additive noise, albedos due to dust and specimen oxidation, artifacts coming from scratches occurring during the specimen preparation. High performance image analysis procedure to distinguish the nodules from the background can be obtained within the framework of image segmentation: the original image is partitioned into disjoint domains where the signal has homogeneous characteristics, and passing from one domain to another these characteristics vary significantly [14]. For the cast iron (and ductile iron) classification it suffices to choose the class of piecewise constant functions to approximate the gray level distribution of the LOM images, so that in the segmented image the nodules are sharply enhanced over a uniform background. Then any standard software can quantify the graphite elements morphological parameters of interest; in particular, the Image Processing Toolbox of MatLabC provided a good performance.

The segmentation problem can be solved by various techniques [15-16] with different pros and cons. The method of active contours [17] was preferred since can deal with the complex topology of the graphite elements without compromising the numerical complexity: as a consequence the cast iron metallographies can be reliably segmented and evaluated in real time.

In this work, different graphite elements morphologies have been considered, ranging from lamellae to nodules, and image segmentation by the active contours method has been optimized in order to perform a quantitative analysis and characterization. A complete automatization of this approach allows to perform statistical analysis of many morphological parameters (e.g., graphite elements density, distribution, shape), allowing to fully characterize the investigated cast iron.

\section{IMAGE SEGMENTATION BY THE ACTIVE CONTOURS METHOD}

$\mathrm{D}$ espite a visual inspection can distinguish the objects from the background on the available real data, the signal is quite irregular and does not feature a clear cut between the background and the different objects of interest in the picture. In Fig. 5-8, some examples of specimens are presented; as it can be noted, in each image there are some scratches and dust and, obviously, graphite elements like exploded graphite and flakes. It is important also to distinguish among the different kind of objects. 


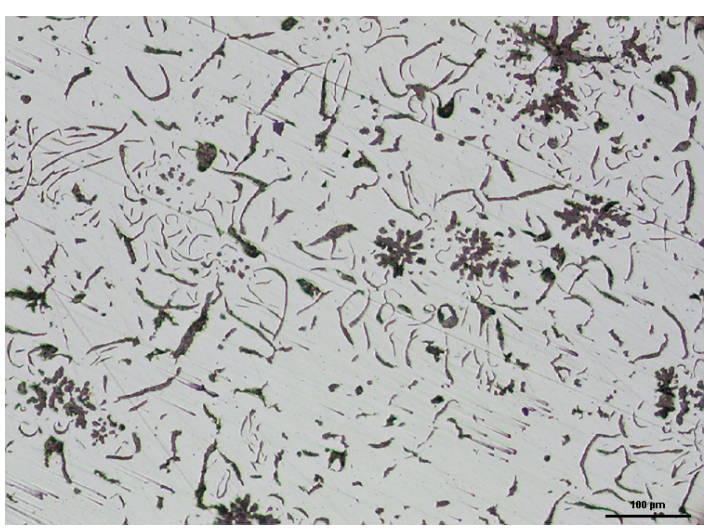

Figure 5: Flakes and irregular spheroidal graphite

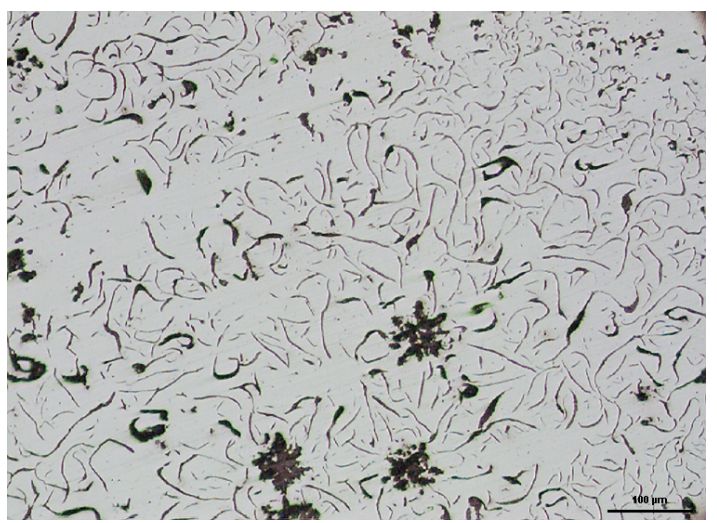

Figure 7: Flakes and slightly irregular spheroidal graphite.

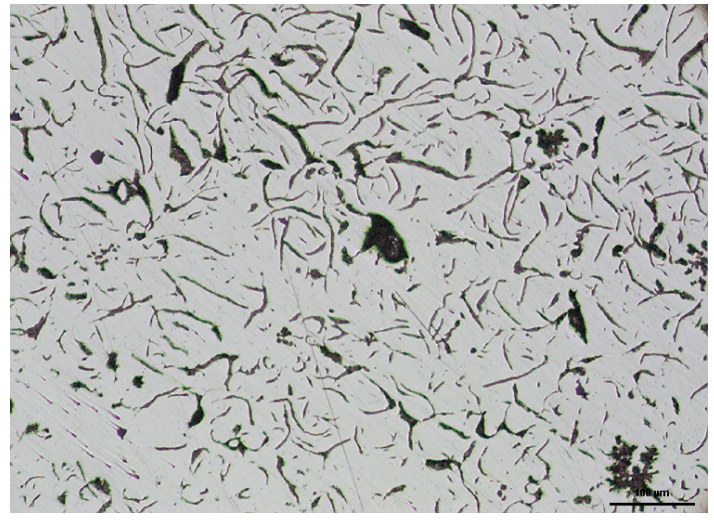

Figure 6: Flakes and compacted (vermicular) graphite.

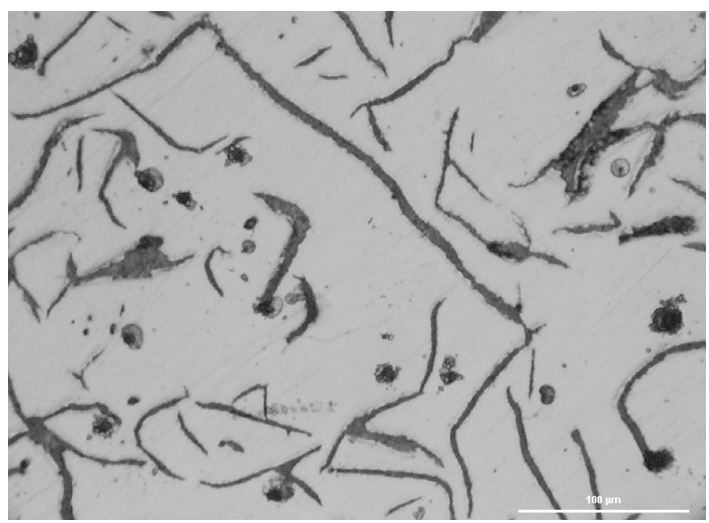

Figure 8: Flakes (higher magnification than Fig. 5-6)

The problem is solved once real data are segmented. The segmentation is the partition of the image in regions homogeneous with respect to some properties, for example the gray level, the shape, the colour, the texture and so on. For the images considered in this paper, a segmentation with respect to the gray level will adequately suit the purpose, obtaining the various objects and the background clearly separated as sub regions of the image domain with constant gray level. The procedure that will be briefly recalled in the following was first proposed in [17] and applied in [18].

Let $\Omega \subset R^{2}$ be a compact subset representing the image domain; the image signal is modeled as a continuous function $g: \Omega \rightarrow[0,1]$. The image segmentation we will refer to is given by

$$
g_{s}=\sum_{i=1}^{N} c_{i} I_{\Omega_{i}}, \quad \Omega=\bigcup_{i=1}^{N} \Omega_{i}, \quad C=\bigcup_{i=1}^{N} \partial \Omega_{i}
$$

where $I_{\Omega_{i}}$ is the characteristic function of set $\Omega_{i}$.

The segmentation problem can be stated as follows:

Given $g: \Omega \rightarrow[0,1]$, find $g_{s}$, that means finding a finite partition $\Omega_{1}, \ldots, \Omega_{N}$ and $c_{1}, \ldots, c_{N}$ such that $g_{s}$ represents $g$ according to some criterion.

The active contour method considered in this work has been suitably adapted to the problem of ductile iron obtaining a very efficient algorithm. Without loss of generality, from now on we will refer to an image with just one object $P$ on the background; we denote by $\Omega_{1}$ the subset of $\Omega$ points corresponding to the object. Therefore $C=\partial \Omega_{1}$ defines the object contour, while $\Omega_{2}=\Omega \backslash \Omega_{1}$ denotes the region outside the object.

The following energy functional $F\left(c_{1}, c_{2}, C\right)$ is assumed:

$$
F\left(c_{1}, c_{2}, C\right)=\mu \cdot \ell(C)+v \cdot A\left(\Omega_{1}\right)+\lambda_{1} \int_{\Omega_{1}}\left|g(x, y)-c_{1}\right|^{2} d x d y+\lambda_{2} \int_{\Omega_{2}}\left|g(x, y)-c_{2}\right|^{2} d x d y
$$


where $\mu \geq 0, v \geq 0, \lambda_{1}, \lambda_{2}>0$ are weights that can be easily fixed, depending on the application. The first two terms represent respectively the length of the object boundary and its area; in the optimization procedure they allow to obtain an optimal solution where $C$ is the union of arcs of regular curves of finite length. The last two terms represent the quadratic approximation error in the set of piecewise constant functions $g_{s}=c_{1} I_{\Omega_{1}}+c_{2} I_{\Omega_{2}}$.

The problem

$$
\min _{c_{1}, c_{2}, C} F\left(c_{1}, c_{2}, C\right)
$$

is well posed in the chosen set up (i.e. piecewise constant functions and piecewise regular boundary $C$ ) where the existence and uniqueness of a global minimum is discussed in [17] and guaranteed in a discrete set up in [19].

According to the level set approach, $C$ is represented by the zero level set of a Lipschitz function $\phi$

$$
C=\{(x, y): \phi(x, y)=0\}
$$

Therefore

$$
\Omega_{1}=\{(x, y): \phi(x, y) \geq 0\}, \quad \Omega_{2}=\{(x, y): \phi(x, y)<0\}
$$

Then, the energy functional can be rewritten as

$$
\begin{aligned}
F\left(c_{1}, c_{2}, \phi\right)= & \mu \int_{\Omega} \delta(\phi(x, y))|| \nabla \phi(x, y)|| d x d y+v \int_{\Omega} H(\phi(x, y)) d x d y \\
& +\lambda_{1} \int_{\Omega}\left|g(x, y)-c_{1}\right|^{2} H(\phi(x, y)) d x d y+\lambda_{2} \int_{\Omega}\left|g(x, y)-c_{2}\right|^{2}(1-H(\phi(x, y))) d x d y
\end{aligned}
$$

where $H(s)$ is the Heaviside function (distribution) and $\delta(s)$ is the Dirac function obtained as (distributional) derivative of $H(s)$

In order to compute the Euler-Lagrange equation for $F\left(c_{1}, c_{2}, \phi\right)$, a smoother version of this functional is considered by substituting to the distributions $H(s)$ and $\delta(s)$ regularized approximants $H_{\varepsilon}(s)$ and $\delta_{\varepsilon}(s)$, obtained as follows

$$
H_{\varepsilon}(s)=\frac{1}{2}\left(1+\frac{2}{\pi} \arctan \left(\frac{s}{\varepsilon}\right)\right), \quad \delta_{\varepsilon}(s)=H_{\varepsilon}^{\prime}(s)=\frac{1}{\pi} \frac{\varepsilon^{2}}{s^{2}+\varepsilon^{2}}
$$

These are $C^{\infty}(\Omega)$ functions and as $\varepsilon \rightarrow 0$ they converge to $H(s)$ and $\delta(s)$ respectively.

Therefore the smooth functional is the following

$$
\begin{aligned}
F_{\varepsilon}\left(c_{1}, c_{2}, \phi\right) & =\mu \int_{\Omega} \delta_{\varepsilon}(\phi(x, y))|\nabla \phi(x, y)| \mid d x d y+v \int_{\Omega} H_{\varepsilon}(\phi(x, y)) d x d y \\
& +\lambda_{1} \int_{\Omega}\left|g(x, y)-c_{1}\right|^{2} H_{\varepsilon}(\phi(x, y)) d x d y+\lambda_{2} \int_{\Omega}\left|g(x, y)-c_{2}\right|^{2}\left(1-H_{\varepsilon}(\phi(x, y))\right) d x d y
\end{aligned}
$$

Optimal solution for constants $c_{1}, c_{2}$ and the equation for $\phi$ are given by the Euler-Lagrange equation:

$$
\begin{aligned}
& c_{1}(\phi)=\frac{\int_{\Omega} g(x, y) H_{\varepsilon}(\phi(x, y)) d x d y}{\int_{\Omega} H_{\varepsilon}(\phi(x, y)) d x d y}, \quad c_{2}(\phi)=\frac{\int_{\Omega} g(x, y)\left(1-H_{\varepsilon}(\phi(x, y))\right) d x d y}{\int_{\Omega}\left(1-H_{\varepsilon}(\phi(x, y))\right) d x d y} \\
& \delta_{\varepsilon}(\phi)\left[\mu \cdot \operatorname{div}\left(\frac{\nabla \phi}{|\nabla \phi|}\right)-v-\lambda_{1}\left(g-c_{1}\right)^{2}+\lambda_{2}\left(g-c_{2}\right)^{2}\right]=0 \quad \text { in } \Omega \\
& \frac{\delta_{\varepsilon}(\phi)}{|\nabla \phi|} \frac{\partial \phi}{\partial \vec{n}}=0 \text { on } \partial \Omega
\end{aligned}
$$

where $\vec{n}$ is the outward normal to $\partial \Omega$ and $\partial \phi / \partial \vec{n}$ is the directional derivative of $\phi$ computed on $\partial \Omega$. 
The (9) and (10) provide a 2-levels segmentation, that is a binarization; if necessary, the procedure may be hierarchically applied and a 2 n-levels segmentation may be determined.

From a numerical point of view, the key feature of the level set approach consists in finding the solution of the previous partial differential non linear equation as steady state solution of the following evolution equation

$$
\begin{aligned}
& \frac{\partial \phi}{\partial t}=\delta_{\varepsilon}(\phi)\left[\mu \cdot \operatorname{div}\left(\frac{\nabla \phi}{|\nabla \phi|}\right)-v-\lambda_{1}\left(g-c_{1}\right)^{2}+\lambda_{2}\left(g-c_{2}\right)^{2}\right] \quad \text { in }(0, \infty) \times \Omega \\
& \phi(0, x, y)=\phi_{0}(x, y) \text { in } \Omega \\
& \frac{\delta_{\varepsilon}(\phi)}{|\nabla \phi|} \frac{\partial \phi}{\partial \vec{n}}=0 \text { on } \partial \Omega
\end{aligned}
$$

where $t$ is a fictitious "time variable"; it is proved that such an equation has a solution $\phi(t, x, y)$ for any $t$, starting from the initial configuration $\phi_{0}$. Moreover $\lim _{t \rightarrow \infty} \phi(t, x, y)=\phi(x, y)$, where $\phi(x, y)$ is the sought solution of the equation $(10)$. As a result we obtain that the zero level set

$$
C(t)=\{(x, y): \phi(t, x, y)=0\}
$$

evolves from the initial arbitrary contour

$$
C(0)=\left\{(x, y): \phi_{0}(x, y)=0\right\}
$$

to

$$
C=\{(x, y): \phi(x, y)=0\}
$$

which is the best representation of the actual object contour according to our optimal criterion.

In Videos 4-7 (see Fig. 9-12) the evolution of the $\mathrm{C}(\mathrm{t})$ curve is shown: at each time $\mathrm{t}$ the curve is positioned at the relative solution of the evolution equation.

More precisely, in order to solve (11) we need to consider that the available data are the samples $\left\{g_{i, j}\right\}$ of function $g$ on a grid of pixel $\left\{\left(x_{i}, y_{j}\right)\right\}$. Therefore (11) is discretized to obtain the samples $\left\{\phi\left(t_{k}, x_{i}, y_{j}\right)\right\}=\left\{\phi_{i, j}{ }^{k}\right\}, t_{k}=k \Delta t$, where $\Delta t$ is the time step. Various approximating schemes can be adopted to solve (11); the one proposed in [19] which leads to following finite difference non linear equation was adopted:

$$
\begin{aligned}
& \frac{\phi_{i, j}{ }^{k+1}-\phi_{i, j}{ }^{k}}{\Delta t}=\delta_{\varepsilon}\left(\phi_{i, j}{ }^{k}\right)\left[\frac { \mu } { ( \Delta b ) ^ { 2 } } \Delta _ { - } ^ { x } \left(\frac{{ }^{\frac{\left(\Delta_{-}^{x} \phi_{i, j}{ }^{k+1}\right)^{2}}{(\Delta b)^{2}}+\frac{\left(\phi_{i, j+1}^{k}-\phi_{i, j-1}^{k}\right)^{2}}{(2 \Delta b)^{2}}}}{(\Delta h)^{2}} \Delta_{-}^{y}\left(\frac{\Delta_{+}^{y+1} \phi_{i, j}{ }^{k+1}}{\sqrt{\frac{\left(\Delta_{+}^{y} \phi_{i, j}{ }^{k+1}\right)^{2}}{(\Delta b)^{2}}+\frac{\left(\phi_{i+1, j}^{k}-\phi_{i-1, j}^{k}\right)^{2}}{(2 \Delta b)^{2}}}}\right)\right.\right. \\
& \left.-v-\lambda_{1}\left(g_{i, j}-c_{1}\left(\phi^{k}\right)\right)^{2}+\lambda_{2}\left(g_{i, j}-c_{2}\left(\phi^{k}\right)\right)^{2}\right]
\end{aligned}
$$


where

$$
\begin{array}{ll}
\Delta_{-}^{x} \phi_{i, j}=\phi_{i, j}-\phi_{i-1, j}, & \Delta_{+}^{x} \phi_{i, j}=\phi_{i+1, j}-\phi_{i, j} \\
\Delta_{-}^{y} \phi_{i, j}=\phi_{i, j}-\phi_{i, j-1}, & \Delta_{+}^{y} \phi_{i, j}=\phi_{i, j+1}-\phi_{i, j}
\end{array}
$$

and $\Delta h$ is the spatial step. The terms $c_{1}\left(\phi^{k}\right), c_{2}\left(\phi^{k}\right)$ are the constants $c_{1}(\phi), c_{2}(\phi)$ computed at $k-t h$ step.

The zero level set evolves (active contour) from the initial shape to the final configuration that well approximates the actual object contour. The evolution ends when the difference in (12) becomes less than a chosen threshold.

\section{NUMERICAL RESULTS: SEGMENTATION AND DATA ANALYSIS}

A s already pointed out, in the segmented image all the objects are well separated, see Fig.9-12 in which the A
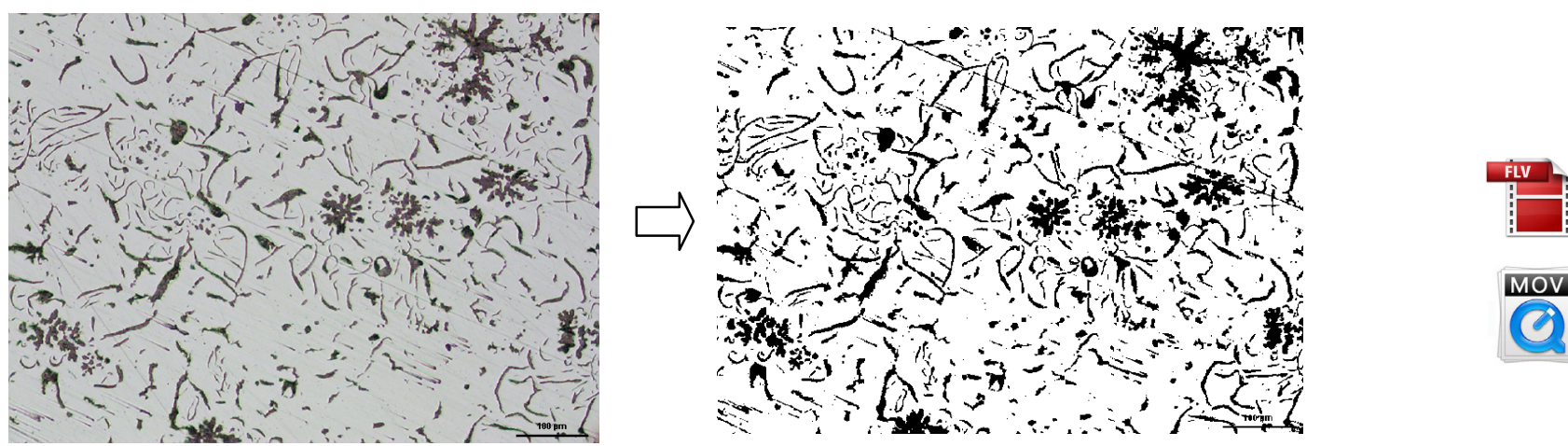

Figure 9: 2-levels segmentation of image of Fig. 5 [V4].
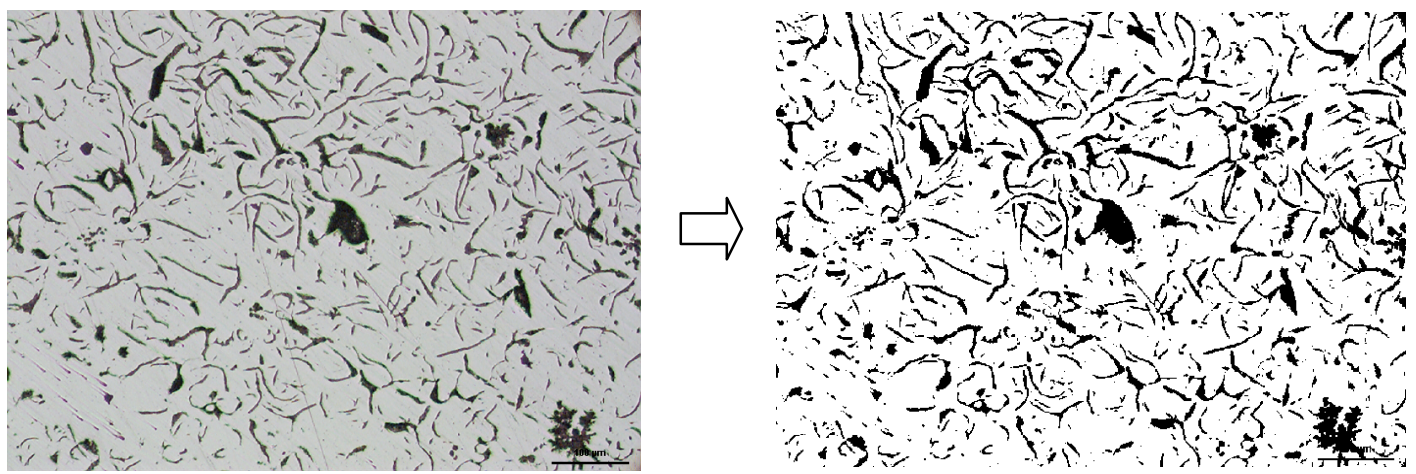

Figure 10: 2-levels segmentation of image of Fig. 6 [V5].
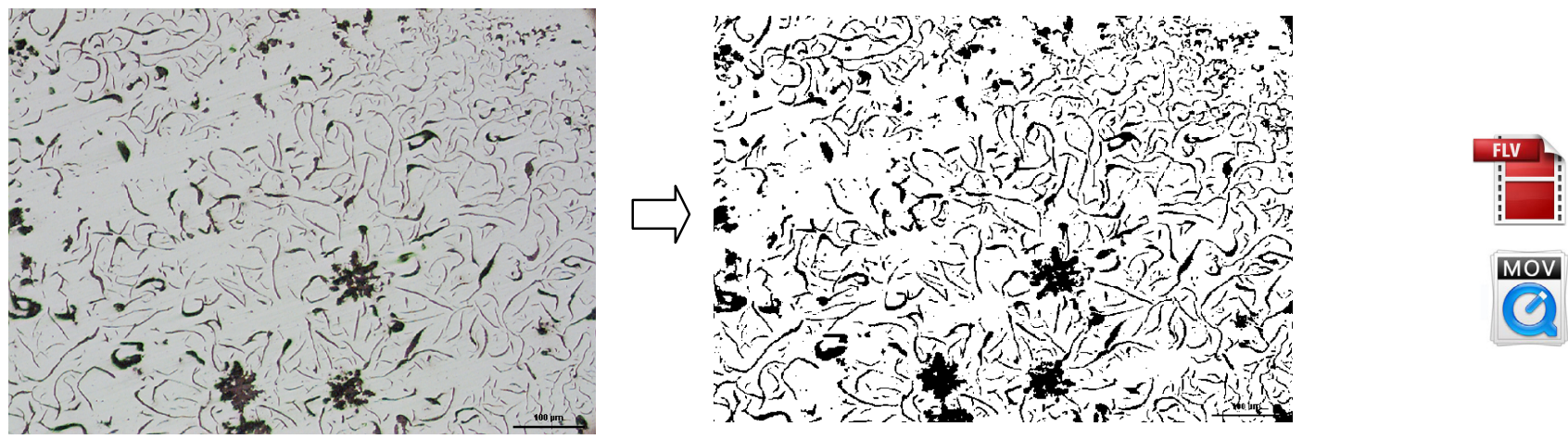

Figure 11: 2-levels segmentation of image of Fig. 7 [V6]. 

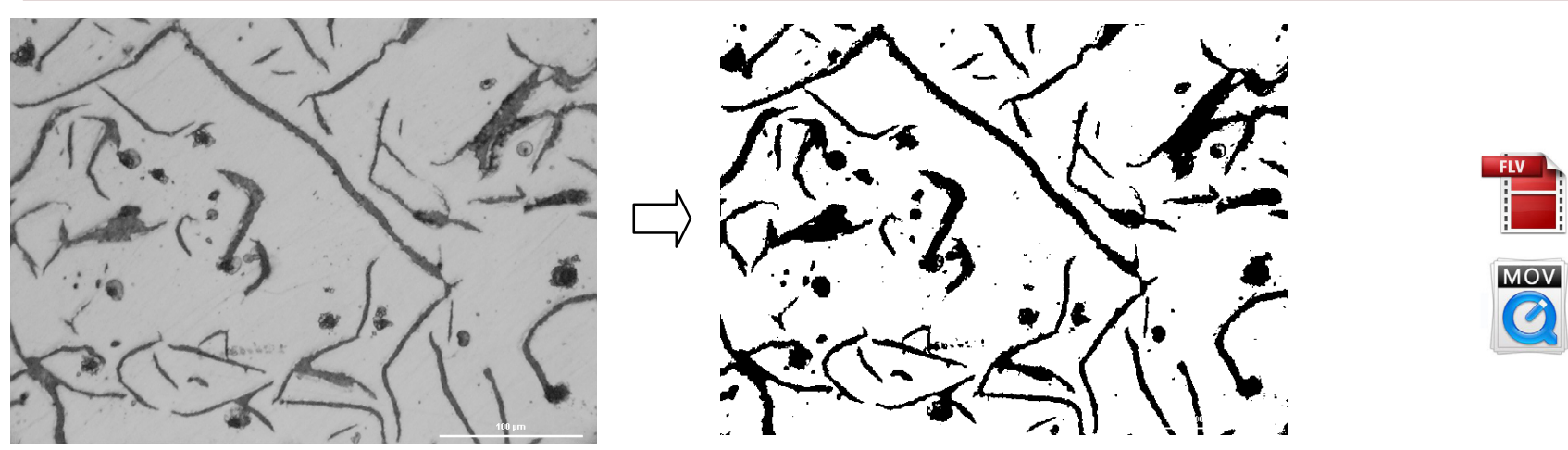

Figure 12: 2-levels segmentation of image of Fig. 8 [V7].

The applied segmentation method is robust with respect to the choice of the parameters in (8). The algorithm used one level set function yielding segmentation with two values $c_{1}, c_{2}$, one for the background and the other for the nodules that were normalized to 1 and 0 ; the algorithm parameters were set to the following values

$$
\mu=1, \quad v=1, \lambda_{1}=\lambda_{2}=10^{5}, \varepsilon=1
$$

This choice has guaranteed a good compromise between segmentation accuracy and rate of convergence: it determines a greater influence of the square approximation error term in functional (2) with respect to the boundary length and area terms.

Once the images are segmented it is possible to determine useful properties from the objects identified versus the background. Any standard software can quantify the elements morphological parameters of interest; in particular, the Image Processing Toolbox of MatLabC provided a good performance.

The morphological features of interest for the purposes of the paper concern: the characteristics of the shape of the spheroids and their position (and, consequently, their distribution) in the specimen.

As far as the shape is concerned, and focusing the ductile irons, it is important to recognize how far is the shape from a circular one and the possible presence of holes inside. For the description of the shape of the spheroids the Area, the Eccentricity and the Solidity parameters may be here considered for the specimen classification:

- the Area of each object identified is evaluated by counting the number of its pixels;

- the Eccentricity is a property of the ellipse that best fits the spheroid: it has values in [ $\left[\begin{array}{ll}0 & 1\end{array}\right]$ and describes how far is the graphite element shape from being circular;

- the Solidity is obtained as the ratio between the Area (number of pixels in the spheroid region) and the ConvexArea (number of pixels in the convex hull). It also takes values in $\left[\begin{array}{ll}0 & 1\end{array}\right]$, the closer to 1 "the more solid" the spheroid: this implies that the spheroid region has a more convex shape, with little ragged contour, and nearly no holes inside. Solidity is closely related to parameter $\mathrm{S}_{\mathrm{AA}}$ defined in [20].

The presence of holes (or "white spots" due to embedded matrix) inside the nodule can be identified by means of the number of Euler: it is defined as the number of objects in the region minus the number of holes in those objects.

Considering other graphite element morphologies (e.g., flaky cast iron) other geometrical properties can be easily determined (e.g., the length of the lamellae).

As far as the position of the objects of interest is concerned, their centroid is available so it is possible to determine, for example, if there is a concentration of nodule in a region or, on the contrary, if there are zones in which no object (nodules or flakes) is present.

As examples of the entire procedure, image in Fig. 13 (and Video 8) is analyzed. A ferritic ductile iron is observed by means of a light optical microscope, considering a high magnification (x1000). The graphite nodule is visually characterized by a reduced nodularity and by the presence of "white spots", probably due to the presence of embedded matrix. Furthermore, in the center of the nodule is also evident an out of focus zone, due to the metallographic procedure and to the lower wear resistance of the nodule core with the respect to the nodule outer shield [6]. It is worth to note that the presence of this out of focus zone does not influence the result. After the binarization, the Image Processing Toolbox of MatLab@ provided the following information:

- the nodule is centered in the point with coordinates $(370,277)$, from the left upper corner;

- the nodule area is constituted by 152977 pixels;

- the nodule eccentricity is 0.43 ; 
- the Euler number is -41 (i.e. in one object minus 42 holes);

- the solidity is 0.94 ;

- the convex area is 163194 ;

- the orientation (i.e. the angle, ranging from -90 to 90 degrees, between the major axis of the ellipse that has the same second moment of the nodule and the horizontal axis) is -88.9.

All these information are in general available for each nodule in an image, allowing statistical considerations that may be useful to characterize the specimens. For example it may be useful to investigate the presence of objects with the same orientation, or if there are nodules particularly deformed (high eccentricity) or with ragged edges (low solidity). These characteristics are strictly related to the mechanical properties of the material, and the statistical analysis may help in the determination of residual life.
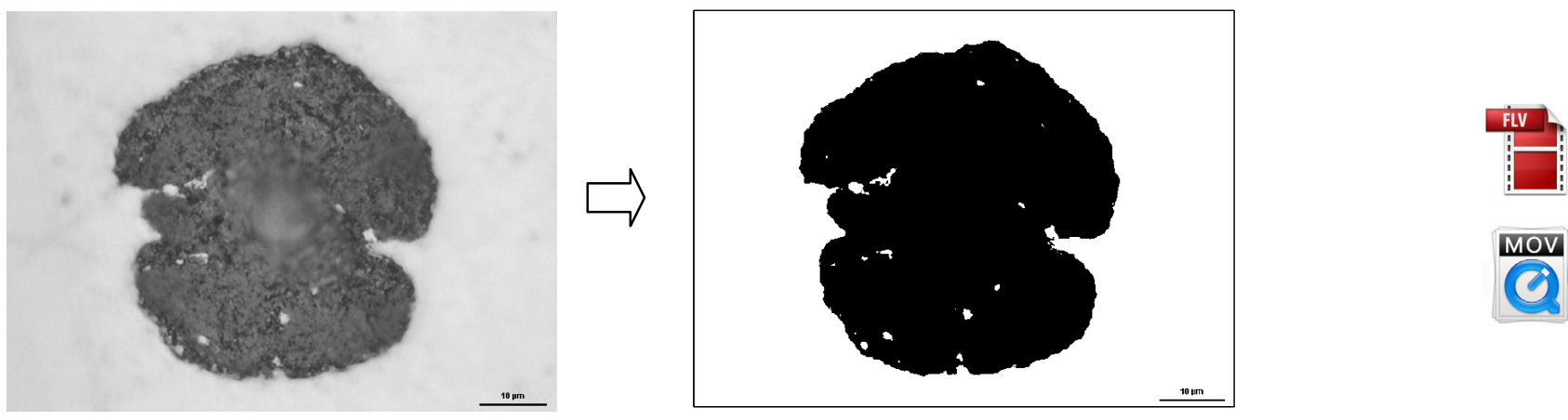

Figure 13: 2-levels segmentation of a graphite nodule (high magnification, 1000x) [V8].

\section{CONCLUSIONS}

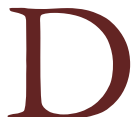

uctile irons mechanical properties and damaging micromechanisms are strongly influenced by the matrix microstructure and by the graphite nodules morphology: a correct evaluation of graphite elements morphology is always the main goal in cast irons microstructural analyses. Graphite elements metallographic analysis standard is based on a visual qualitative approach, that does not seem to be sufficient to completely characterize the graphite elements morphology parameters (mainly, but not only, shape, dimension, distribution).

In this work, the image segmentation by the active contours method is optimized in order to be applied on cast iron: this approach allows a quantitative analysis of the graphite elements, ranging from flakes to nodular ones. After the application of active contours method, it is possible to define many morphological parameters, both considering low magnification images (and focusing parameters like elements density and distribution) and high magnification images, allowing to quantitatively analyze every single graphite element.

\section{REFERENCES}

[1] Iacoviello, F., Di Cocco, V., Piacente, V., Di Bartolomeo, O., Damage micromechanisms in ferritic-pearlitic ductile cast irons, Mater. Science and Engng. A, 478 (2008) 181-186.

[2] Di Cocco, V., Iacoviello, F., Cavallini, M., Damaging micromechanisms characterization of a ferritic ductile cast iron, Engineering Fracture Mechanics, 77 (2010) 2016-2023.

[3] Iacoviello, F., Di Cocco, V., Cavallini, M., Ductile cast irons: microstructure influence on fatigue crack propagation resistance, Frattura ed Integrità Strutturale, 13 (2010) 3-16.

[4] Cavallini, M., Di Cocco, V., Iacoviello, F., Iacoviello, D., in: G. Ferro, F. Iacoviello, L. Susmel (Eds), XXI Convegno Nazionale del Gruppo Italiano Frattura (IGF), Cassino, Italy, (2011) 415-414.

[5] Di Cocco, V., Iacoviello, F., Rossi, A., Cavallini, M., Natali, S., in: F. Iacoviello, G. Risitano, L. Susmel (Eds), Acta Fracturae, Gruppo Italiano Frattura (IGF), Rome, Italy, (2013) 1-8.

[6] Di Cocco, V., Iacoviello, F., Rossi, A., Cavallini, M., Natali, S., Graphite nodules and fatigue crack propagation micromechanisms in a ferritic ductile cast iron, Fatigue Fract Engng Mater Struct (FFEMS), 36(9), (2013) 893-902. 
[7] Dong, M.J., Hu, G. K., Diboine, A., Moulin, D., Prioul, C., Damage modelling in nodular cast iron, Journal de physique IV, 03 (1993) C7-643-C7-648

[8] Dong, M.J., Berdin, C., Beranger, A.S., Prioul, C., Damage effect in the fracture toughness of nodular cast iron, Journal de physique IV, 6 (1996) C6-65-C6-74.

[9] Dong, M.J., Prioul, C., François, D., Damage effect on the fracture toughness of nodular cast iron: Part I. Damage characterization and plastic flow stress modelling, Metall. And Mater. Trans. A, 28A (1997) 2245.

[10] Berdin, C., Dong, M.J., Prioul, C., Local approach of damage and fracture toughness for nodular cast iron, Engineering Fracture Mechanics, 68 (2001) 1107-1117.

[11] Di Cocco, V., Iacoviello, F., Rossi, A., Cavallini, M., Natali, S., Ecarla, F, in: F. Iacoviello, G. Risitano, L. Susmel (Eds), Acta Fracturae, Gruppo Italiano Frattura (IGF), Rome, Italy, (2013) 222-230.

[12] Lundberg, M., Calmunger, M., Lin Peng, R., in: Proceedings of $13^{\text {th }}$ International Conference on Fracture, Beijing, China (2013).

[13] EN ISO 945-1 (2009) Standard.

[14] R. C. Gonzalez, R. E. Woods, Digital Image Processing, Prentice Hall, New Jersey, (2002).

[15] Zuva, T., Olugbara, O.O., Ojo, S.O., Ngwira, S.M., Image segmentation, available techniques, developments and open issues, Canadian Journal on Image Processing and Computer Vision, (2011), 2 (3), 20-29.

[16] Saini, R., Dutta, M., Kumar, R., A comparative study of several image segmentation techniques, Journal of Information and Operations Managements, (2012) 3 (1), 21-24.

[17] Chan, T.F., Vese, L., Active contours without edges. IEEE Trans. Image Proc. 10, (2001) 266-277

[18] De Santis, A., Di Bartolomeo, O., Iacoviello, D., Iacoviello, F., Quantitative shape evaluation of graphite particles in ductile iron, J. of Materials Processing Technology, 196 (1-3) (2008) 292-302.

[19] De Santis, A., Iacoviello, D., Discrete level set approach to image segmentation, Signal, Image and Video Processing, Springer-Verlag London, (2007) 1(4): 303-320.

[20] ASTM Standard A445-63T; (1985) Annual Book.

\section{VIDEO REFERENCES}

[V1] http://www.gruppofrattura.it/video/FIS26/Iacoviello/V1.mp4

[V2] http://www.gruppofrattura.it/video/FIS26/Iacoviello/V2.mp4

[V3] http://www.gruppofrattura.it/video/FIS26/Iacoviello/V3.mp4

[V4] http://www.gruppofrattura.it/video/FIS26/Iacoviello/V4.mp4

[V5] http://www.gruppofrattura.it/video/FIS26/Iacoviello/V5.mp4

[V6] http://www.gruppofrattura.it/video/FIS26/Iacoviello/V6.mp4

[V7] http://www.gruppofrattura.it/video/FIS26/Iacoviello/V7.mp4

[V8] http://www.gruppofrattura.it/video/FIS26/Iacoviello/V8.mp4 